\title{
Trends in ambulatory cardiology consultations for suspected myocarditis after COVID-19 vaccination
}

\author{
Holger Eggebrecht ${ }^{1} \cdot$ Philipp Breitbart $^{1} \cdot$ Alexander Koch $^{1} \cdot$ Bernd Nowak $^{1} \cdot$ Claudia Walther $^{1} \cdot$ Thomas Voigtländer $^{1}$. \\ Christoph Liebetrau' ${ }^{1}$ Rajendra H. Metha ${ }^{2} \cdot$ Axel Schmermund $^{1}$
}

Received: 27 September 2021 / Accepted: 16 November 2021 / Published online: 23 November 2021

(c) The Author(s), under exclusive licence to Springer-Verlag GmbH Germany 2021

Sirs:

In Germany, vaccination against coronavirus disease 2019 (COVID-19) was approved on December 21st 2020 and the first patient treated on December 26th. On May 7th 2021, the European Medicines Agency (EMA) announced an official investigation of case reports of myopericarditis in temporal relation to COVID-19 vaccination [1-3], which was updated on June 11th [4]. Since then, we noted increasing requests from General Practitioners for urgent cardiologic consultation of patients with suspected myocarditis after COVID-19 vaccination. All patients were scheduled within the 2 following business days and underwent standardized cardiologic assessment including electrocardiography (ECG), echocardiography, and determination of NT-proBNP and highsensitive troponin T. Cardiac magnetic resonance (CMR) was performed at the discretion of the treating cardiologist. Statistical analysis was performed using IBM SPSS Statistics 27.0. Continuous variables are presented as mean and $95 \%$ confidence interval $[\mathrm{CI}]$, and categorical variables are represented as frequencies and percentages. Comparisons were performed with Student's $t$ test and with the Chi-square test for categorical variables. A two-sided $p$ value of $\leq 0.05$ was considered statistically significant.

Between December 27, 2020 and September 3, 2021, a total of 113 patients (age: 45.9 years, 95\% CI 43.2-48.7; $44 \%$ females) were referred to our cardiology practice for suspected myopericarditis after COVID-19 vaccination. Cumulative number of patients increased exponentially after the EMA investigation (Fig. 1). Forty-nine (43\%) patients

Holger Eggebrecht

h.eggebrecht@ccb.de

1 Cardioangiological Center Bethanien (CCB), Im Prüfling 23, 60389 Frankfurt, Germany

2 Duke Clinical Research Institute (DCRI) and Duke University Medical Center, Durham, NC, USA presented after the first vaccine dose (delay: 25.5 days, $95 \%$ CI 18.8-32.1), while the remaining 64 (57\%) patients were referred after the second dose (28.6 days; 95\% CI 20.7-36.5). Vaccines included BNT162b2 mRNA (Pfizer-BioNTech) $(n=85,75 \%)$, mRNA-1273 (Moderna) $(n=13,12 \%)$, ChAdOx1 nCoV-19 (AstraZeneca) $(n=5,4 \%)$, and Ad26. COV2.S (Johnson\&Johnson) $(n=6,5 \%)$. Four patients (4\%) received a combination (ChAdOx $1 \mathrm{nCoV}-19$, followed by BNT162b2 mRNA). Symptoms reported after vaccination that triggered evaluation included chest pain $(n=68,60 \%)$, shortness of breath $(n=36,32 \%)$, palpitations $(n=41,36 \%)$, fatigue $(n=25,22 \%)$, and/or reduced exercise tolerability $(n=30,27 \%)$.

In 96/113 (85\%) patients, myopericarditis was ruled out on the basis of normal ECG, echocardiography, and lab values. In 17 patients, additional CMR was performed for persistent suspicion of myopericarditis. In four of them, CMR ultimately confirmed myopericarditis. These patients presented with ST elevations $(n=1)$, ST depressions $(n=1)$, raised troponin $(n=2)$, and/or mild pericardial effusion on echocardiography $(n=2)$. Patients with myopericarditis were younger compared to those without (30.5 vs. 46.5 years, $\mathrm{p}=0.033)$, and had higher levels of troponin T (192 vs. $4.7 \mathrm{pg} / \mathrm{ml} ; p<0.001)$ and NT-proBNP (149 vs. $79 \mathrm{pg} / \mathrm{ml}$; $p=0.342)$. There were no differences in left-ventricular ejection fraction (LVEF) $(67.0 \%$ vs. $65.8 \%, p=0.683)$ or leftventricular (LV) end-diastolic dimensions ( 45.8 vs $47.7 \mathrm{~mm}$, $p=0.399$ ). In the four patients with myopericarditis, CMR showed evidence myocardial injury by subepicardial late gadolinium enhancement as well as evidence of myocardial edema by T1/2 mapping and minor pericardial effusion, but LV function (65.5\%, 95\% CI 61.2-68.9) and cardiac dimensions were also within normal range. During follow-up (59.3 days; 95\% CI 33.5-85.0), symptoms of myopericarditis patients improved $(n=1)$ or resolved $(n=3)$. Troponin and NT-proBNP were normal in all patients at follow-up. 


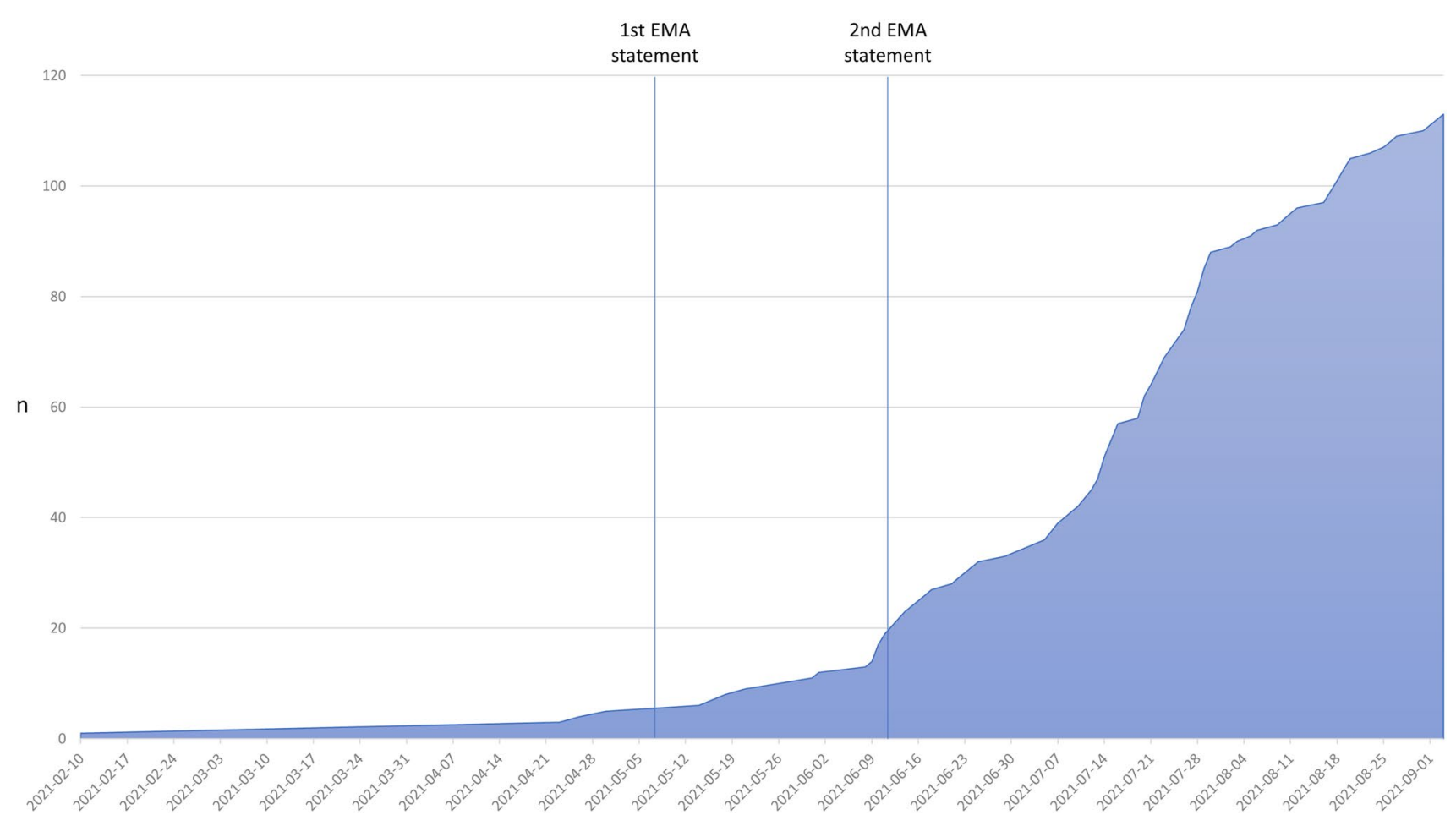

Fig. 1 Cumulative number of patients with suspected myopericarditis after COVID-19 vaccination. Lines depict the dates of the EMA investigation announcements

CMR showed stable LVEF (64.3\% vs. $62.5 \%$ at baseline) with regressive edema.

In the setting of an ambulatory cardiology practice, numbers of patients referred for suspected myopericarditis after COVID-19 vaccination increased significantly. However, confirmed myopericarditis in temporal relation to COVID19 vaccination was rare and involved only 4 out of 113 symptomatic patients (Table 1). In all four patients, CMR depicted mild myocardial injury with normal LVEF. During short-term follow-up, no deterioration of LV function and rapid clinical recovery were observed. Given the increasing number of vaccine recipient reporting cardiac symptoms and rarity of confirmed myopericarditis makes routine use of CMR for diagnosis of myocarditis post-COVID-19 vaccination cost-prohibitive. However, particularly in symptomatic young boys and adolescents who carry a significantly increased risk of myopericarditis after mRNA vaccination [2], CMR remains important to rule out vaccine-associated myocardial injury. 
Table 1 Patient characteristics

Myopericarditis confirmed $(n=4) \quad$ No evidence of myo- $p$ pericarditis $(n=109)$

\begin{tabular}{|c|c|c|c|}
\hline Age (years) & $30.5(14.0-46.9)$ & $46.5(43.8-49.2)$ & 0.033 \\
\hline Female gender $(\%)$ & $2(50 \%)$ & 48 & 0.814 \\
\hline \multicolumn{4}{|l|}{ Vaccine } \\
\hline BNT162b2 mRNA & $2(50 \%)$ & $83(76 \%)$ & \multirow[t]{5}{*}{0.182} \\
\hline mRNA-1273 & $2(50 \%)$ & $11(10 \%)$ & \\
\hline ChAdOx1 nCoV-19 & 0 & $5(5 \%)$ & \\
\hline Ad26.COV2.S & 0 & $6(6 \%)$ & \\
\hline Combination $\mathrm{ChAdOx}+\mathrm{BNT}$ & 0 & $4(4 \%)$ & \\
\hline Only 1 st dose received & $3(75 \%)$ & $61(56 \%)$ & 0.451 \\
\hline 2nd dose received & $1(25 \%)$ & $48(44 \%)$ & 0.451 \\
\hline Days since last vaccine dose received & $14.8(4.6-24.9)$ & $27.7(22.2-33.2)$ & 0.370 \\
\hline \multicolumn{4}{|l|}{ Symptoms } \\
\hline Chest pain & $3(75 \%)$ & $65(60 \%)$ & 0.537 \\
\hline Shortness of breath & $1(25 \%)$ & $35(32 \%)$ & 0.764 \\
\hline Palpitations & $1(25 \%)$ & $40(37 \%)$ & 0.633 \\
\hline Fatigue & $1(25 \%)$ & $24(22 \%)$ & 0.888 \\
\hline Reduced exercise tolerability & $1(25 \%)$ & $29(27 \%)$ & 0.943 \\
\hline Abnormal ECG (\%) & $2(50 \%)$ & $9(8 \%)$ & 0.006 \\
\hline Echocardiographic LVEF (\%) & $67.7(59.6-75.7)$ & $65.8(64.8-66.9)$ & 0.683 \\
\hline Pericardial effusion $(\%)$ & 0 & $2(2 \%)$ & 1.000 \\
\hline NT-proBNP (pg/ml) & $149(98.6-199.4)$ & $78.2(52.0-104.4)$ & $<0.001$ \\
\hline Troponin T (pg/ml) & $192(-105.1$ to 489.1$)$ & $4.7(4.1-5.3)$ & 0.342 \\
\hline \multicolumn{4}{|l|}{ CMR results } \\
\hline $\operatorname{LVEF}(\%)$ & $65.5,61.2-68.9$ & NA & - \\
\hline Subepicardial LGE $(n)$ & $4 / 4$ & & \\
\hline \multirow[t]{4}{*}{ Mean T1 values (ms) } & Patient 1: 1047 & & \\
\hline & Patient 2: 1053 & & \\
\hline & Patient 3: 1023 & & \\
\hline & Patient 4: 1010 & & \\
\hline \multirow[t]{4}{*}{ Mean T2 values (ms) } & Patient 1: 57 & & \\
\hline & Patient 2: 53 & & \\
\hline & Patient 3: 48 & & \\
\hline & Patient 4: 47 & & \\
\hline Pericardial effusion & 4/4 (minor) & & \\
\hline
\end{tabular}

\section{Declarations}

Conflict of interest On behalf of all authors, the corresponding author states that there is no conflict of interest.

\section{References}

1. Verma AK, Lavine KJ, Lin C (2021) Myocarditis after COVID19 mRNA Vaccination. N Engl J Med. https://doi.org/10.1056/ NEJMc2109975 (Online ahead of print)
2. Bozkurt B, Kamat I, Hotez PJ (2021) Myocarditis with COVID-19 mRNA vaccines. Circulation 144(6):471-484. https://doi.org/10. 1161/circulationaha.121.056135 (Epub 2021 Jul 20)

3. Ehrlich P, Klingel K, Ohlmann-Knafo S, Hüttinger S, Sood N, Pickuth D, Kindermann M (2021) Biopsy-proven lymphocytic myocarditis following first mRNA COVID-19 vaccination in a 40-year-old male: case report. Clin Res Cardiol 6:1-5

4. https://www.ema.europa.eu/en/news/meeting-highlights-pharm acovigilance-risk-assessment-committee-prac-3-6-may-2021, Last visited: 27 Sep 2021 